\title{
Das mystische Erlebnis bei Plotin
}

\author{
Von H. LUDIN JANSEN
}

Wenn auch Plotin in der griechischen Philosophie wohl bewandert war, vor allem natürlich im Platonismus und Stoizismus, und durch Studien und unmittelbare Verbindung zugleich mit Astrologie, Gnostizismus und Mysterienglauben vertraut war, ist es seine persönliche religiöse Erfahrung, die seiner Rede die eigenartige Form und den eigentümlichen Inhalt gibt. Hinter allem, was er uns vorlegt, unterscheiden wir das mystische Erlebnis und sein Bedürfnis, die dort gewonnenen Erfahrungen lebendig darzustellen.

In seiner Biographie Plotins (Kap. 23) erzählt Porphyrios, dass Plotin im Laufe der Zeit, da Porphyrios bei ihm war, vier derartige Erlebnisse gehabt habe. Nun wissen wir aus einer andern Stelle in der Biographie (Kap. 5), dass Porphyrios sechs Jahre mit seinem Lehrer zusammen lebte. Plotin hatte mit andern Worten vier solche Erlebnisse im Laufe von sechs Jahren. Es könnten also Jahre zwischen jedem vergehen. Es ist natürlich, Porphyrios dahin zu deuten, dass er hier an besonders tiefgreifende Erfahrungen denkt. Es ist wohl auch natürlich anzunehmen, dass er Erlebnisse weniger eingreifender Natur zu erwähnen unterlässt. Wenn er ausserdem erklärt, Gott sei oft, $\pi 0 \lambda \lambda \alpha \dot{\alpha}<\iota \varsigma$, dem Plotin erschienen, ist es wohl denkbar, dass dies sich auf solche kleinere Erfahrungen bezieht. Eine andere Möglichkeit ist es, dieses $\pi 0 \lambda \lambda \dot{\alpha} x u \varsigma$ im Verhältnis zu dem, was Porphyrios von sich selber sagt, zu verstehen, er habe in seinem Leben nur eine Erfahrung dieser Art gehabt, obgleich er 68 Jahre war, als er die Biographie schrieb. Im Vergleich damit hatte Plotin viele Erlebnisse.

Plotin ist keine gute Quelle, wenn es gilt die Form seiner Erfahrungen zu erkennen oder die Methode, deren er sich bediente um dieselben zu erlangen. Er interessiert sich in erster Reihe dafür, den religiösen Gedankeninhalt des Erlebnisses klarzulegen, und wir sind daher genötigt, den Stoff, den wir brauchen, in dieser seiner theoretischen Darstellung zu finden. Von Zeit zu Zeit gibt er uns indessen einen Schimmer von dem, was in der Stunde der Vorbereitung und der Entzückung in seinem Gemüte vorging, und eben dieses Material wollen wir verwenden. 
Eine von den Stellen, wo Plotin sein Erlebnis ziemlich ausführlich beschreibt, ist in der Einleitung zum Traktat „Vom Herabsteigen der Seele in die Körper", IV. 8, wo es heisst:

„Oft wenn ich aus meinem Leibe aufwache und frei werde, von all dem Äussern gelöst werde und mich in meinen Sinn versenke, erlebe ich eine ganz wunderbare Schönheit. Da kommt mir ein starkes Gefühl, an einem besseren Leben teilzuhaben, diese höchste Form des Lebens zu verwirklichen und mit dem Göttlichen eins zu werden. Wenn ich aber dann, nachdem ich mich in dem Göttlichen befunden habe und diese Verwirklichung erreicht habe, im Bewusstsein, über alles andere in der intelligiblen Welt erhoben zu sein, wenn ich nach diesem Aufenthalt in dem Göttlichen, von der Welt des Geistes wieder in die Welt des Gedankens herabsteige, da muss ich mich wirklich fragen, wie dieses Herabsteigen möglich sei, und wie die Seele überhaupt in den Leib geraten sei, die Seele, die trotz ihres Aufenthalts im Leibe ein solches Wesen hat, in dem sie mir jetzt erschienen ist."

Diese Schilderung enthält mehrere interessante Momente. Wir merken uns unter anderem den Gedanken, dass das Erlebnis ,ein Aufwachen des

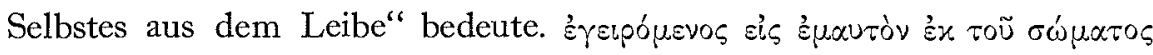
Es ist auch, wie er sagt, eine Begegnung mit einer wunderbaren Schönheit,

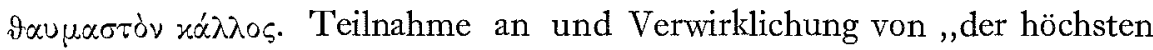

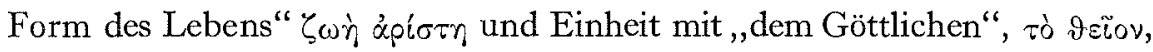
ein Gefühl, erhoben zu sein über ,,alles andere in der intelligiblen Welt", $\dot{j} \pi \dot{\varepsilon} \rho$

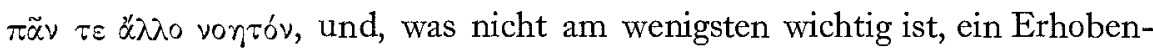
sein über die Welt des Denkens. Nach dem Aufenthalt in dem Göttlichen fiel er zurück, wie er es ausdrückt, von der Welt des Geistes ,in die Welt

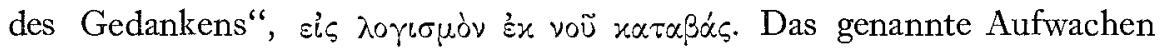
des Selbstes ist, wie wir sehen werden, mehr eine Voraussetzung des Erlebnisses als eine Folge davon. Das ergibt sich übrigens aus dem Wortlaut:

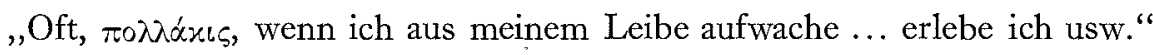
Das Erlebnis erfolgt also nicht immer, sondern oft. Die Stelle scheint etwas anzudeuten, womit wir von andern Mystikern vertraut sind, dass auch Plotin ein vorbereitetes Erlebnis nicht immer gelungen ist.

Plotin sieht es als etwas Wesentliches an, dass er in dieser Begegnung die

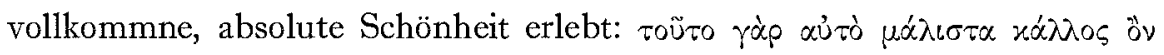

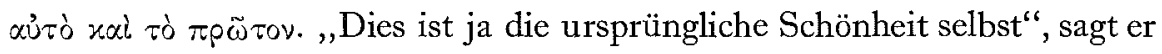


an einer Stelle, I. 6, 7, oder wie er es auch ausdrückt: Es ist ,, herrliches

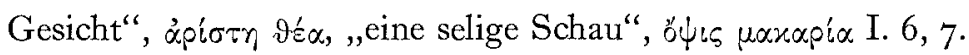

An unserer Stelle ist es von einem Einswerden mit dem Göttlichen die

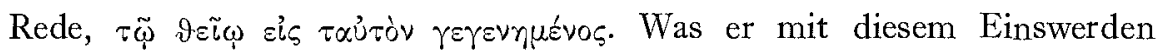
meint, geht an einer andern Stelle deutlicher hervor, wo es heisst:

„Es gab nicht zwei, aber der Schauende war eins mit dem Geschauten, als ob es sich nicht um ein Schauen drehte, sondern um Einheit. Darum muss jeder, der von dieser Mischung geprägt worden ist, ein Bild davon bewahren. Er ist ja selbst eins geworden ( $(v)$ und hat nichts Trennendes, weder in seinem Innern noch im Verhältnis zu seinen Umgebungen. Nichts bewegt ihn mehr in dem Zustand, worin er sich befindet, keine Leidenschaft, kein Verlangen, auch kein Denken, Verstehen noch Selbstbewusstsein, wenn man es so sagen kann. Wie entrückt oder von Gott erfüllt befindet er sich in Ruhe, ohne Bewegung, ohne sich mit seinem Wesen gegen irgend etwas zu beugen oder um sein eigenes Selbst zu kreisen, ganz still, selbst eine vollkommene

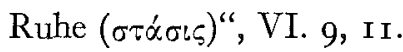

Die Begegnung bedeutet m. a. W. ein völliges Aufgeben von dem Selbst. Er denkt nicht länger, versteht nicht länger, ja, es ist überhaupt nicht mehr

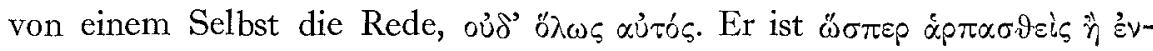
ఫovoí́์ $\sigma \varsigma$ gleichsam entrückt oder von Gott erfüllt. Plotin sagt übrigens hier dasselbe wie im ersten Stück, das wir gelesen haben (IV. 8), er habe sich im Göttlichen befunden oder da geruht. Die Begegnung kann denn als eine Ruhe im Göttlichen angesehen werden.

Jedes Gefühl, ein Mensch zu sein und einen Leib zu haben, verschwindet bei dieser Begegung: „Wenn die Seele plötzlich dieses in sich auflodern sieht, da gibt es nichts zwischen ihr und dem, was sie sieht. Die beiden sind eins ... und sie hat nicht mehr eine Fühlung mit dem Leib; sie kann sich auch nicht auf andere Weise charakterisieren als Menschen, Lebewesen, etwas Seiendes oder alles", VII. 7, 34. Bezeichnend ist das, was hier steht, dass das Gesicht

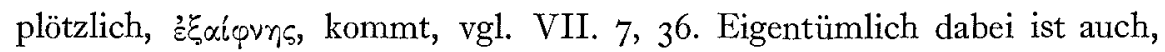
dass es nicht von Ermüdung begleitet wird. Man kann es unbegrenzt genies-

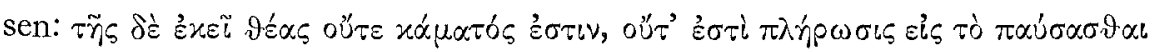

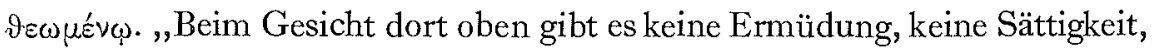
welche für den Schauenden zur Unterbrechung führt, "V. 8, 4 .

Wie für die meisten Mystiker gilt es auch für Plotin, dass dieses Aufgehen 
in die Gottheit und das Verschwinden des Selbstes nicht das einzige Ziel ist. Auch er schillert in seinem Denken zwischen der Idee vom Auslöschen des Individuums und dessen fortgesetztem Bestehen. Letzteres kommt in folgender Aussage deutlich zum Ausdrück: ,Wir müssen darum schnell von hier eilen und uns widerwillig an andere Dinge gebunden fühlen, dass wir mit unserem ganzen Selbst das Jenseitige ergreifen können und keinen 'Teil in uns sein lassen, der nicht Gott berührt. Da kann man droben sowohl ihn als sich selbst sehen dürfen, soweit solche Gesichte denn möglich sind, sich selbst im Strahlenglanz, von geistigem Licht erfüllt, oder sagen wir vielleicht wie reines Licht, ganz leicht, ohne Gewicht, vergottet oder richtiger

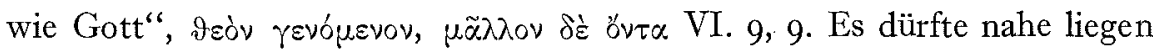
anzunehmen, dass Plotin hier wie in manchen anderen Fällen sein Bild aus dem Mysterium holt.

Von grossem Interesse für uns ist es etwas näher zu betrachten, wie Plotin seine Erlebnisse vorbereitet. Darüber spricht er teils in allgemeineren Wendungen von einer Reinigung des Sinnes, teils in mehr besonderen Aussagen von den konkreten U̇bungen, die nötig sind. Die allgemeine Charakterestik finden wir vor allem in seinem Traktat „Von den Tugenden", I. 2. Es verhält sich nämlich so, dass Plotin etwas ganz Bestimmtes meint, wenn er von Tugenden wie Weisheit, Tapferkeit, Selbsterkenntnis und Gerechtigkeit spricht. Er gebraucht zwar dieselben Termini wie z. B. Platon, legt aber etwas anderes in diese Worte. Weisheit ist ihm der Wunsch der Seele, sich von dem Körper zu entfernen. Tapferkeit bedeutet, dass man diese Trennung nicht fürchtet. Selbsterkenntnis ist die Unberührtheit der Seele von den Forderungen des Körpers, und gerecht ist die Seele, die den Geist walten lässt, I. 6, 6. Mit andern Worten, die Tugenden bestehen in der-

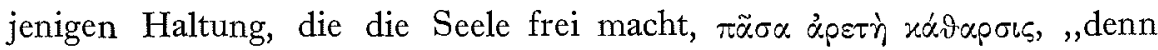
jede Tugend ist Reinigung", I. 6, 6. Tugend in der Bedeutung von bürgerlicher Tugend liegt ausserhalb seines Zielpunkts, wenn er auch derselben einen relativen Wert beimessen kan, z. B. wenn er sagt, dass die icio

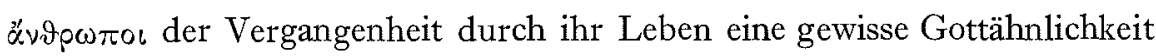
erreichten, I. 2, I.

Alles, was Plotin über Tugend und ethisches Verhalten sagt, dreht sich in der Tat um die Befreiung der Seele, welche die erste Bedingung ist, dass das ekstatische Erlebnis zustande kommen kann. Meister Eckehart spricht, wie 
bekannt, von der Abgeschiedenheit, also von einem Verschlossensein der äusseren Welt gegenüber. Plotin meint dasselbe, wenn er sagt, man solle in das Allerheiligste gehen und, was das Auge sieht, hinter sich lassen, है $\xi_{\omega} \omega$

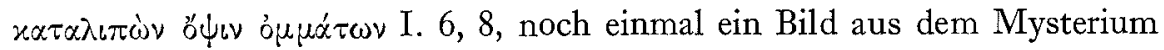
geholt.

Die konkreteren Beschreibungen gelten, wie schon gesagt, die geistigen Übungen, die Plotin für nötig hält. Nachdem diese Abgeschiedenheit nach aussen zustande gekommen ist, muss man nach seiner Ansicht systematisch vorwärts gehen, um weiter zu kommen. Man muss anfangen mit einer Konzentration des Gedankens um ein bestimmtes äusseres Thema, und zwar das Schöne und Gute, das einem im Umgang mit anderen Menschen begegnet ist, jedoch nicht die äusseren Werke, die sie gemacht haben, sondern die Schönheit und Güte der Seele, von denen ihre Werke zeugen, I. 6, 9. Es gilt mit andern Worten den Sinn zu sammeln um das Schöne und das Gute an sich, und dafür zu sorgen, dass der Sinn dies Gesicht festhalte.

Wenn man so weit gelangt ist, wird die nächste Stufe, sich selbst zu erforschen im Lichte von diesem Schönen und Guten. Diese Forschung vergleicht er dann mit dem, was in der Werkstätte des Bildhauers geschieht: „Entdeckt man, dass man noch nicht schön ist, muss man wie der Bildhauer tun, der ein schönes Bild machen will. Bald haut er etwas weg, bald reibt er, bald glättet oder putzt er, bis das schöne Angesicht seines Bildes endlich hervortritt. In derselben Weise müssen auch wir das Überflüssige entfernen, was schief ist, ebnen, das Schmutzige waschen und reinigen, und ohne Aufhören unser eigenes Bild bauen, bis die göttliche Pracht der Tugend hervorstrahlt und wir sehen, dass die Selbstbeherrschung auf heiligem und sicherem Grunde ruht. Wenn man derart geworden ist und sich selber so sieht, bedeutet es, dass man ganz allein ist, und dass es kein Hindernis gibt, dass man als eine Einheit hervortreten kann ... Wenn man sieht dass man so geworden ist, hat man schon das Vermögen zu schauen bekommen und die volle $\mathrm{Zu}$ versicht zu sich selber, so dass man sich erheben kann. Man bedarf keinen Wegweiser mehr, aber kann schauen, wenn man will", I. 6, 9.

Die Seele ist m. a. W. bereit. Was nun geschieht, ist dass die intelligible Welt, voü , sich öffnet, so dass die Seele die vollkommene Schönheit schauen darf. Das Denken hat noch nicht aufgehört, aber es ist nicht länger die Seele, die denkt. Es ist voũs, der denkt und von sich selber spricht. Die Seele 
empfängt nur: „So lange wir uns droben in der Welt der Vernunft (

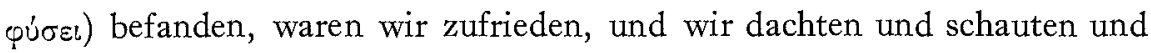
sammelten alles in eins, denn es war voüs, der dachte und von sich selber sprach. Die Seele war in Ruhe und fügte sich dem Wirken des Geistes", V. 3, 6. Noch ist das Selbstbewusstsein lebendig, wenn auch eine passivere, empfangende Haltung in die Seele eingetreten ist. In diesem Moment geschieht es dann, dass das Gesicht erscheint, plötzlich, ohne Vorzeichen: „Das Gesicht kommt plötzlich. Man weiss nicht wie. Es füllt die Augen mit Licht, und wegen dieses Lichtes kann man nichts anderes erblicken. Das einzige, was man erblickt, ist dieses Licht. Man sieht kein Objekt mehr und kein Licht ausserhalb des Objekts; es gibt kein Intellekt und gar kein Objekt des Intellektes. Nein, man sieht nur den Glanz, der so beschaffen ist, dass er erzeugt ohne sich zu entleeren und bleibt ohne sich zu ändern", VI. 7, $3^{6}$.

Das Gesicht hat den Charakter eines starken, glänzenden Lichts, $\varphi \tilde{\omega} \varsigma$, das derart ist, dass alles andre verschwindet, ja, was mehr ist, der Schauende selbst wird zu diesem Licht verwandelt, und sein Gefühl, ausserhalb des Geschauten zu stehen, verschwindet. Objekt und Subjekt fliessen zusammen. Er ist, was er sieht.

Plotin denkt auch darüber nach, wer solche Erlebnisse bekommen kann. Zwar verhält es sich so, dass alle das Vermögen besitzen, aber nur wenige, sagt er, machen davon Gebrauch. „Um zu sehen soll man die Augen schliessen und so das andere Gesicht erwecken, das alle besitzen, aber wenige ge-

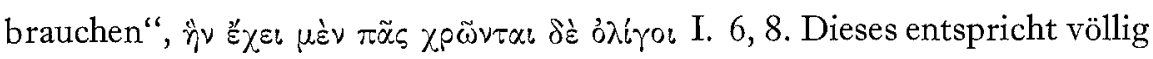
der anthropologischen Idee Plotins, dass der Mensch in sich eine Seele trage, die ihren Ursprung in der Transzendenz habe, und sich dahin zurücksehne,

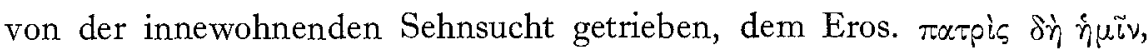

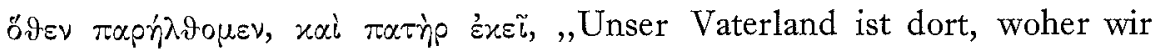
gekommen sind, und da ist unser Vater", I. 6, 8. Dieses Vermögen gebraucht

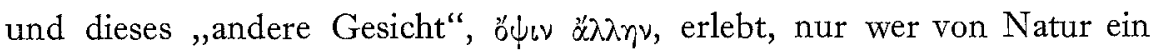

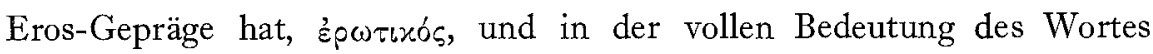
Philosoph ist, ,wer von Anfang an wirklich ein Philosoph von Ausrüstung

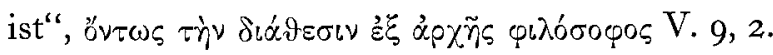

Plotin ist in ausgesprochenem Grade ein intellektueller Mystiker. Das ergibt sich teils aus der Tatsache, dass er in seiner Vertiefung das Intellekt herrschen lässt, um gewisse Motive seinen Gedanken konzentriert, bis der 
Moment kommt, da die Seele ruhig wird, und alles Denken aufhört, teils daraus, dass er in seiner Beschreibung des Erlebnisses dasselbe rationalisert und seinen Inhalt denkend ausseinandersetzt. Der gefühlsmässige, poetische Einschlag in seinen Beschreibungen ist immer seiner strengen Logik und seinem klaren Gedanken untergeordnet. Er ist jedoch darauf aufmerksam, dass es unmöglich ist, das Erlebnis lebendig darzustellen, wenn man es mit Gedanken auseinandersetzt. Da greift er zu demselben Ausweg, wie es alle Mystiker tun, wenn sie fühlen, wie hoffnungslos der Versuch ist, das Un-

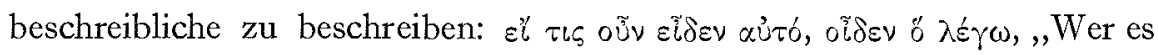
gesehen hat, weiss was ich meine", I. 6, 7. 